University of Nebraska - Lincoln

DigitalCommons@University of Nebraska - Lincoln

Faculty Publications, Department of Psychology

Psychology, Department of

July 2003

\title{
Doing Rawls Justice: An Experimental Study of Income Distribution Norms
}

Philip A. Michelbach

University of California, San Diego

John T. Scott

University of California, Davis

Richard E. Matland

University of Houston

Brian H. Bornstein

University of Nebraska-Lincoln, bbornstein2@unl.edu

Follow this and additional works at: https://digitalcommons.unl.edu/psychfacpub

Part of the Psychiatry and Psychology Commons

Michelbach, Philip A.; Scott, John T.; Matland, Richard E.; and Bornstein, Brian H., "Doing Rawls Justice: An Experimental Study of Income Distribution Norms" (2003). Faculty Publications, Department of Psychology. 186.

https://digitalcommons.unl.edu/psychfacpub/186

This Article is brought to you for free and open access by the Psychology, Department of at DigitalCommons@University of Nebraska - Lincoln. It has been accepted for inclusion in Faculty Publications, Department of Psychology by an authorized administrator of DigitalCommons@University of Nebraska - Lincoln. 


\title{
Doing Rawls Justice: An Experimental Study of Income Distribution Norms
}

\author{
Philip A. Michelbach University of California, San Diego \\ John T. Scott University of California, Davis \\ Richard E. Matland University of Houston \\ Brian H. Bornstein University of Nebraska-Lincoln
}

\begin{abstract}
Distributive justice has been the focus of political theory with the postwar rise of the social welfare state, and Rawls'A Theory of Justice (1971) is arguably the most important work of political philosophy during that period. Parallel to this theoretical literature is a body of empirical research into distributive justice. We offer a synthesis of the theoretical and empirical approaches with an experimental study of how individuals use allocation principles in making judgments concerning income distribution under conditions of strict impartiality. Our experiment is designed in part to examine the extent to which they prioritize them consistent with Rawls' theory. We find that distributive justice judgments are complex but structured, with individuals tending to use several principles simultaneously and weighing them according to predictable factors, with sex and race being particularly important. We also find that individuals use several strategies in using competing allocation principles and that a considerable minority prioritize them consistent with a Rawlsian maximin strategy.
\end{abstract}

$\mathrm{D}$ istributive justice has been the focus of normative political theory over the last half century, and John Rawls' A Theory of Justice (1971) is widely seen as the most important attempt during that period to articulate a set of institutions and distributional outcomes that rational individuals would see as legitimate. Rawls' seminal work has spawned a veritable critical industry since its publication (Daniels 1989; Miller 1999). His recent elaboration of his project and restatement of his theory of "justice as fairness" $(1996,2001)$ promise to sustain interest in his ideas.

Rawls' project of constructing a situation in which impartial individuals choose principles of justice may resonate particularly strongly for members of the modern social welfare state because we expect similarly impartial behavior and fairness in the distribution of social benefits and burdens. Indeed, research suggests the legitimacy of the state depends in large measure on perceived procedural and distributive justice (Rasinski
1987; Weatherford 1992; Alwin, Gornev, and Khakhulina 1995). Empirical researchers are therefore interested in people's views on distributive justice, but the picture emerging from public opinion and other studies is one of individuals who seem to have conflicting views (Lane 1962; Hochshild 1981; McClosky and Zaller 1984; Verba and Orren 1985; Kluegel and Smith 1986; Verba et al. 1987; Kluegel, Mason, and Wegener 1995). Experimental research nonetheless reveals that people have complex rather than conflicting ideas about justice (see Miller 1999, chaps. 3-4; Törnblom 1992; Elster 1995; Tyler et al. 1997; Hegtvedt and Cook 2001). As we recently argued (Scott et al. 2001), distributive justice behavior is complex but structured: distributive justice judgments involve several distinct allocation principles and are influenced in predictable ways by independent factors. The experimental method is an ideal way to critically analyze theories of justice (see Elster 1995) and to explore the structure of distributive justice behavior, both to un-

Philip A. Michelbach, Ph.D. Candidate, Department of Political Science, University of California, San Diego, La Jolla, CA 92093-0521 (pmichelb@weber.ucsd. edu). John T. Scott, Associate Professor of Political Science, University of California, Davis, Davis, CA 956168682 (jtscott@ucdavis.edu). Richard E. Matland, Associate Professor of Political Science, University of Houston, Houston, TX 77204347 (matland@uh.edu). Brian H. Bornstein, Associate Professor of Psychology, University of Nebraska-Lincoln, Lincoln, NE 68588 (bbornstein2@unl.edu).

This study was funded by research grants from the National Science Foundation (SBR-9810243 and SBR-9810473) and a Faculty Research Grant from the University of California, Davis. We would like to thank Pauline Schloesser for help in data collection and Richard J. Arneson, Gary W. Cox, Benjamin Highton, Brian R. Sala, and James F. Spriggs II for their comments and suggestions. 
derstand a broad range of social phenomena and to design proper instruments to study them in all their complexity (see Sniderman et al. 1986; Lacy 2001).

This article synthesizes normative and empirical research with an experimental study of how individuals use allocation principles in judgments of income distribution under conditions of strict impartiality. Strictly impartial individuals are the focus for two reasons. First, we want to understand distributive behaviors and institutions that are-or are supposed to be-impartial in nature. Second, we are interested in the relationship of the normative principles themselves apart from any instrumental factors, since, despite the focus on solely instrumental theories in much social science research, numerous studies show that normative beliefs also determine people's behavior (see Sen 1977; Tyler et al. 1997). As Konow (2001, 139; see 2000) argues, we need to isolate "unbiased justice" to understand the interactions among egocentric and principled factors that determine behavior. Our experiment is designed in part to explore the results of our study concerning gender differences in distributive justice behavior and to extend their analysis to race (Scott et al. 2001). In order to permit a meaningful test of the influence of race in particular, we use a large and diverse participant pool. Our experiment is also specifically designed to examine to what extent individuals use a Rawlsian maximin strategy in prioritizing allocation principles, enabling us to explore the contradictory results of recent experimental studies (Frohlich and Oppenheimer 1992; Mitchell et al. 1993).

We begin with a discussion of prior research, outlining the allocation principles we examine in relation to Rawls' theory of justice and reviewing previous experimental studies. Next we present the experimental design and our theoretical predictions. We then present the results of two separate analyses: a regression analyses of individuals' behavior and a categorization of strategies individuals' use in prioritizing allocation principles according to several theoretical models. Finally, we offer a discussion of our results and conclude with some observations on the complex structure of distributive justice behavior and its importance for social inquiry.

\section{Allocation Principles in Distributive Justice Research}

While there are myriad theories about what is "just" or "fair," there is also considerable consensus in theoretical and empirical research over a small number of allocation principles. Four analytically distinct allocation principles emerge from a review of the literature: equal- ity, efficiency, need, and merit. Since our experiment is designed in part to determine to what extent individuals prioritize these principles consistent with Rawls' expectations, we will outline them with reference to his theory of justice.

\section{Allocation Principles in Rawls' Theory of Justice}

Rawls' aim is to construct a theory of justice by establishing how rational individuals would choose principles of justice under a condition of strict impartiality. He calls this approach "justice as fairness" $(1971,11)$. He proposes a thought experiment in which he asks what principles would be chosen behind a "veil of ignorance" where individuals do not know their social standing, attributes, etc. He makes reference in his resulting theory to all four of the allocation principles we have identified. Rawls acknowledges that individuals in actuality very probably do make justice decisions by balancing several potentially competing allocation principles and using their intuitions to assign relative weight. While other prominent theories of justice adopt such an "intuitionist" or "pluralist" approach (e.g., Walzer 1983; Miller 1999), Rawls rejects it as inadequate and instead embarks on a "constructivist" project in which he argues that these principles should be ordered insofar as possible (1971, 34-45).

Rawls suggests that individuals in the original position would first choose equality of basic political and social rights. Equality also plays an important role in his specifically distributive theory, for absolute equality of income is the a priori privileged distributional outcome and therefore "the benchmark for judging improvements" (1971, 60, 65, 100-1). While there is considerable disagreement over exactly what equality means (Rae 1981; Sen 1992), we follow Rawls and most other modern theories of justice by defining equality in absolute terms. Despite the primacy of equality, Rawls argues that inequality of income and other social goods can be justified by a concern for increased productivity, or efficien$c y$. Like Rawls, we use the term "efficiency" to mean that individuals prefer a greater amount of overall goods for the same amount of input (see Sen 1992, esp. 6-8). Raw1s terms the tension between efficiency and equality the "aggregative-distributive dichotomy" (1971, 36, 44; see Okun 1975). He argues that after establishing equality of opportunity, rational individuals would tolerate inequality only to the extent that any increased efficiency benefits everyone, and especially "the least well-off."

The "difference principle," as Rawls terms this justification of inequality, reveals a concern for need. Rawls has been increasingly clear in framing the difference 
principle in these terms $(1971,277 ; 1996$, esp. 187-90). One difficulty facing theoretical development of need as a distinct normative principle is the strong conceptual relationship between need and equality, especially when need is conceived of as relative rather than absolute (see Braybrooke 1987; Miller 1999, 203-30). To distinguish the two principles, we consider need in absolute terms as a minimum level of necessary social goods, in keeping with Rawls' concept of "social minimum."

Finally, the principle of merit plays a crucial role in Rawls' theory by way of exclusion. He argues that individuals not only do not deserve the advantages they enjoy from wealth, connections, and other privileges, but that they similarly do not deserve any advantages from the "natural lottery," such as intelligence, beauty, strength, or even the desire to work hard (1971, 15, 73-4). According to Rawls, the unequal results these advantages produce in terms of increased efficiency should be permitted only for their beneficial consequences to the neediest. Some theorists agree with Rawls concerning the moral irrelevance of merit (e.g., Nozick 1974; Okun 1975; Hayek 1976), but others follow a tradition dating back at least to Aristotle by maintaining that merit is a legitimate allocation principle (e.g., Sandel 1982; Sher 1987; Miller 1999). Our experiment is designed to determine whether individuals do find merit morally relevant and how it affects their use of other allocation principles.

\section{Experimental Research} on Distributive Justice

Three previous studies are particularly relevant for our research. The first two studies are designed to test Rawls' predictions, but produce contradictory results. Frohlich and Oppenheimer (1992) find almost no support among experimental subjects for a Rawlsian maximin model. In their experiments, participants in groups are asked to determine rules for distributing income they are to earn by doing an unspecified task (behind a "veil of ignorance"). Of the four alternative models among which groups choose, they find that groups overwhelmingly choose maximizing income after setting a minimum "floor" income (78\%) and almost never choose a Rawlsian solution (1\%).

In contrast, Mitchell et al. (1993) argue that their study reveals considerable support for Rawls. They ask individual participants acting as advisors to a hypothetical society to rank income distributions posing tradeoffs between equality and efficiency. The major experimental manipulation is to vary what participants are told about the degree to which merit explains income in that society. They suggest their results support Rawls in two ways. First, they argue Rawls' suspicion about the validity of merit is confirmed since participants tend to prefer equality over efficiency when luck and other "morally arbitrary" factors are said overwhelmingly to determine income. The finding nevertheless suggests only that individuals may act consistent with Rawls' expectations under certain conditions. Second, they find the behavior of a significant number of participants fits their stipulated Rawlsian maximin model. This interpretation is nonetheless questionable because their a priori model specification may not distinguish egalitarian and Rawlsian solutions since their measure of equality is the absolute difference between income quartiles rather than the proportional difference (Scott et al. 2001, 752). Although the measurement of inequality is a complex and often highly technical subject (see Temkin 1993; Sen 1997), all major measures are proportional rather than absolute, and we therefore use a proportional measure in our experiments. Finally, since Mitchell et al. (1993) do not vary need independent of equality it is not clear whether their participants were intolerant of inequality or sensitive to need. Later work by two of the authors of their study points out this flaw and shows that individuals exhibit distinct concern for need (Ordónez and Mellers 1993).

Finally, our recent study (Scott et al. 2001) examines how individuals use all four allocation principles by varying them independently. We find that equality, efficiency, merit, and need all play a distinct role in distributive justice judgments and that most individuals use all or most of these principles simultaneously (see also Konow 2000, 2001). We also uncover an unexpectedly strong gender difference in this behavior. Aside from finding that women are more egalitarian than men, we find that women's preferences for equality and efficiency are affected by information about merit, with women being less concerned with equality as merit increasingly predicts income, but that men's behavior is not affected by such information. One of our principal aims in the present study is to explore and extend our results by using an altered experimental design and expanded participant pool. Finally, another design difference enables us to distinguish a Rawlsian maximin solution from other egalitarian and efficiency-maximizing strategies in order to examine the contradictory findings of previous studies.

\section{Experimental Design and Theoretical Predictions}

Our experiment examines how individuals use allocation principles in judgments of income distribution under conditions of strict impartiality. In this section we out- 
line our experimental design and discuss how it enables us to test hypotheses about distributive justice behavior through two types of analyses: regression analysis of between-subject behavior and categorization of withinsubject use of allocation principles according to theoretical models, including a Rawlsian maximin model.

\section{Experimental Presentation}

Participants read a short description of a hypothetical society and are asked to imagine they are outside observers giving their advice to the society. In order to insure impartiality they are explicitly told they are not members of this society. They will evaluate different policies being considered by the society based on the effects they will have on the level and distribution of income as determined by "economic studies" (the policies themselves are not described). They are then presented with a sample income distribution like those they will evaluate (see Figure 1) along with instructions on how to interpret it. (See appendix for participant instructions.) After taking a short manipulation check, described below, they evaluate nine randomly ordered income distributions. As in the sample income distribution, each distribution consists of a bar graph that shows the average income for each quintile and the poverty line and presents information about the overall average income and the ratio of the incomes of the top and bottom quintiles (the "income inequality ratio"). Participants evaluate each distribution on a scale from 0 ("very bad") to 10 ("very good"). Finally, they complete a post-experimental questionnaire that elicits information used for the control variables.

The manipulation check that participants take after reading the country description and before rating the income distributions is designed to ensure the internal

\section{FIGURE 1 Sample Income Distribution}

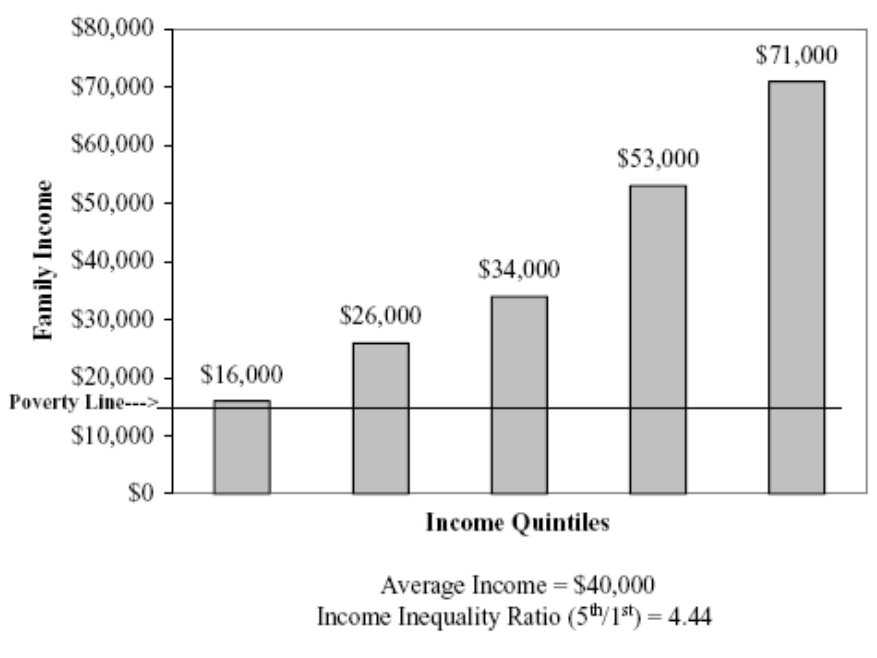

ing that participants have been successfully manipulated by the merit and need variables and questions that ascertain whether they can correctly interpret the information about efficiency and equality in the income distributions. Participants who do not answer all these questions correctly or do not complete the experiment are not included in the data set. We can therefore be as confident as possible that participants are revealing meaningful information about their preferences under the different experimental conditions.

\section{Experimental Manipulations}

The income distributions rated by participants (Table 1) are created by manipulating two variables: efficiency and equality. We operationalize efficiency as average societal income and vary it in three levels (low, medium, high): $\$ 32,000, \$ 42,000$, and $\$ 55,000 .{ }^{1}$ We operationalize equality as the ratio between the highest and lowest income quintiles of an income distribution (the "income inequality ratio") and vary it in three equality levels (high, medium, low). The range of income inequality for the nine distributions is 2.0 for the most equal to 9.0 for the least equal. We increase income inequality in approximately equal steps across the three efficiency levels within each equality level, with two exceptions described below.

Varying equality and efficiency in this way poses participants with equality-efficiency tradeoffs that enable us to determine the relative weight they place on the two principles. Each tradeoff reveals whether participants prefer greater efficiency at the cost of lower equality or greater equality at the cost of lower efficiency. The most conspicuous equality-efficiency tradeoffs are the four diagonal cases, represented by the solid arrows along the diagonals in Table 1. For example, there is a tradeoff between the high-equality/medium-efficiency distribution and the medium-equality/high-efficiency one. The former provides moderate average income but a fairly equal overall distribution, while the latter provides significantly greater income overall but with greater income inequality.

In addition to posing participants with equality-efficiency tradeoffs, this design presents them with tradeoffs between Rawlsian and egalitarian preferences. In order to create those tradeoffs we allow two exceptions to the general rule of increasing the inequality of distribu-

${ }^{1}$ U.S. Census Bureau data was used to construct distributions. In our experiment the middle efficiency level is $\$ 42,000$ for the average income of a four-person family. Median income for a four-person family for 1998 was $\$ 42,709$ (http://www.census. gov/hhes/income/ 4person.html). 


\section{TABLE 1 Experimental Income Distributions}

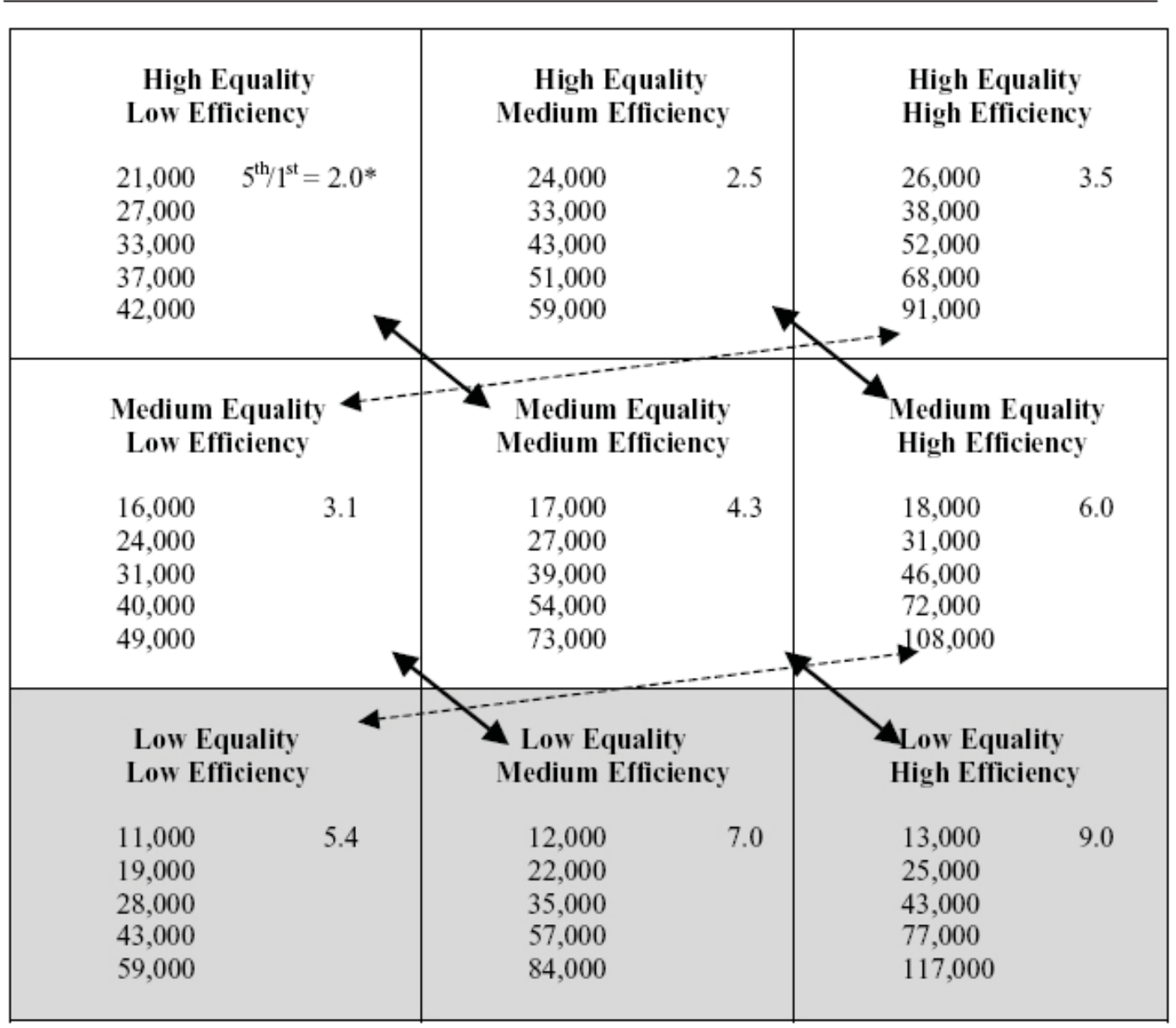

*Income inequality ratio: $5^{\text {th }}$ quintile $/ 1^{\text {st }}$ quintile

tions in approximately equal steps within each equality level by placing a higher equality-income distribution in the lower equality level. These inverted cases are indicated by the dashed arrows in Table 1. For example, the medium-equality/low-efficiency distribution would be the preferred outcome for strong egalitarians, because it has a lower inequality level, while the high-equality/ high-efficiency distribution would be the preferred outcome for Rawlsians, because increased efficiency provides greater income for those with the lowest income. We will take advantage of this design innovation in our second general analysis when we create theoretical models to categorize participants' observed behavior.

Two variables manipulated between participants allow us to study the relationship between equality-efficiency preferences and the other two allocation principles: merit and need. First, we randomly vary what participants are told about the degree to which income is explained by effort and ability (10\%, 50\%, and 90\%), as opposed to luck, connections, and similar factors, in order to examine how equality-efficiency preferences are affected by assumptions about merit. Second, we randomly vary what participants are told about the poverty line $(\$ 10,000$ or $\$ 15,000)$ in order to distinguish participants' sensitivity to need from their desire for equality. ${ }^{2}$ The poverty line manipulation affects the income distributions in the low-equality level, or the bottom (shaded) row in Table 1. When the poverty line is $\$ 10,000$, the lowest income quintile in each of the three low-equality income distributions is above the poverty line, but when the poverty line is $\$ 15,000$, these same quintiles are below the poverty line, making need a distinct issue.

${ }^{2}$ U.S. Census Bureau data was used in designing the poverty manipulation. The poverty line for a four-person family in 1997 was \$16,400 (http://www.census.gov/hhes/poverty/threshld97.html). 


\section{Experimental Setting and Participants}

Participants are undergraduates at the University of Houston and Texas Southern University. Participants from the University of Houston were tested in undergraduate courses in political science and sociology, while participants from Texas Southern University were tested in undergraduate political science courses. There are 667 total participants in the data set (397 women and 270 men), with a diverse population (291 whites, 140 blacks, 142 Hispanics, 58 Asians, and 36 other race). There are no statistically significant differences among testing sites or groups, so we combine the data for all analyses.

\section{Theoretical Predictions and Construction of Variables}

Our theoretical predictions apply to both our regression analyses of between-subject behavior and our categorization of within-subject use of allocation principles, but the discussion of the construction of variables pertains specifically to the regression analyses. We will present theoretical models of the use of allocation principles based on our design when we turn to the categorization of participant behavior.

Equality-efficiency preferences. Our manipulation of equality and efficiency levels in the income distributions is designed to examine how participants use equality and efficiency as allocation principles, especially in relation to one another. We examine participants' use of equality and efficiency in both our general analyses. First, in our regression analysis we examine how equality-efficiency preferences and need sensitivity interact with assumptions about merit and how they are affected by independent variables such as gender and race. Our dependent variable is an Equality-efficiency tradeoff score created by summing the differences between a participant's ratings of the two distributions in each of the four diagonal tradeoffs indicated by the solid arrows in Table 1 (e.g., high equality/low efficiency - medium equality/medium efficiency). Preferences for equality result in higher (positive) scores, while preferences for efficiency produce lower (negative) scores. Second, we further examine participants' use of egalitarian and efficiency-maximizing strategies in our categorization of participant behavior according to different theoretical models. We also develop a distinct Rawlsian maximin model in this analysis to determine to what extent participants use equality and efficiency in accordance with Raw1s' theory. Based on previous research (esp. Mitchell et al. 1993; Scott et al. 2001), we anticipate that relatively few participants will be "pure" types, either strong egalitarians, strong efficiency maximizers, or Rawlsians. Instead, we expect that most participants will use both equality and efficiency as allocation principles and make tradeoffs between them with varying emphases on the two principles and different strategies for weighing them.

Need sensitivity. The poverty-line manipulation examines the distinctness of need as an allocation principle. In the regression analyses, a Poverty dummy variable identifies the poverty condition to which a participant is randomly assigned: 0 if the participant is in the $\$ 10,000$ group and 1 in the $\$ 15,000$ group, where some distributions are affected by the poverty line. We examine need sensitivity in our regression analyses with the dependent variable being a Need sensitivity score created by summing a participant's ratings of the three distributions affected by the poverty manipulation, i.e., the three low-equality distributions in the bottom (shaded) row in Table 1. Higher scores indicate decreased sensitivity to need. We expect that participants will give lower ratings to distributions affected by the poverty manipulation, with the poverty dummy variable therefore having a negative effect on the need-sensitivity score. This result would be consistent with several studies showing a distinct concern for need (Scott et al. 2001; Frohlich and Oppenheimer 1992; Ordónez and Mellers 1993).

Merit effects. The merit manipulation is designed to test whether equality-efficiency preferences and need sensitivity are mediated by assumptions about the degree to which income is explained by effort and ability, or "merited." We examine the effect of merit in our regression analyses, where we treat the merit level as a trichotomous variable. The merit factor $(0,1,2)$ identifies the merit condition to which a participant is randomly assigned $(10 \%, 50 \%$, and $90 \%$, respectively). We expect that participants will be less concerned with equality as the degree to which income is merited increases. The merit factor should therefore have a negative effect on the equality-efficiency tradeoff variable. As noted above, although we found that information about merit had a statistically significant effect on the equality-efficiency preferences of women but not men (Scott et al. 2001), we anticipate that our design change may reveal the same effect for both genders. We suggested that the failure of information about merit to have a significant effect among men might have been because the tradeoffs between equality and efficiency were so modest as not to trigger a reaction from them given their strong preference for efficiency. In the present study we increase the 
cost of trading off equality for efficiency by using a wider range of income inequality (2.0 to 9.0 compared to 2.0 to 6.1 for the earlier study) to see whether men are affected by merit assumption. Finally, we also anticipate that merit may have a negative effect on the need sensitivity variable since participants may view those below the poverty line as increasingly "deservedly" poor. This finding would be consistent with the tradeoff Ordónez and Mellers (1993) find between need and merit, as well as our earlier study, although we found this effect for females alone.

Control variable effects. Our post-experimental questionnaire elicits information on demographic and political values that provide measures for the control variables that studies have suggested affect distributive justice judgments. Of primary interest to us given previous research is gender. A number of studies show that women have a greater preference for equality, while men tend to weigh efficiency more heavily (Gilligan 1982; Major and Deaux 1982; Major and Adams 1983; Kluegel and Smith 1986, chap. 5; Scott et al. 2001). In addition, women have been found to be more sensitive to need and concerned with poverty (Kluegel and Miyano 1995; Davidson, Steinmann and Wegener 1995; Scott et al. 2001). We therefore predict that being female will have a positive effect on the equality-efficiency tradeoff score and a negative effect on the need sensitivity score. As noted earlier, our previous study showed that this gender effect is associated with the merit manipulation (Scott et al. 2001), and we employ an interaction term for gender and merit in our regression analyses to explore this connection.

Race is also a potentially important factor, although there is very little relevant experimental research to give us guidance in making predictions. As noted previously, because of the size of our sample and the diversity of the population at our testing sites we have sufficient numbers of minority participants to look at this population separately. Recent research on issues of distributive justice has found that blacks generally endorse income disparity based on merit beliefs (Hochschild 1995). Other research has suggested that differences between blacks and whites over social policies are due in large measure to differences over fundamental principles, such as equality of opportunity (Kinder and Winter 2001), or alternatively are reflections of differences in group interest (Jackman 1994). Our expectation is that minority respondents will show a greater desire for equality and will be more sensitive to issues of need. To test this we create a dummy variable for minority participants and also include an interaction term for this variable with the experimental manipulations we employ (merit and poverty) to explore the possible differential effects of those experimental manipulations on these participants.

Finally, we include a question designed to test the internal validity of our experimental manipulations. To test for the possibility that participants may be relying on their own perceptions of the degree to which merit explains income our own society rather than our experimental manipulation of merit level, in the post-experimental questionnaire we ask participants for their own view on the degree to which income is predicted by effort and ability in the U.S., or their independent merit perception (0-100\%). If the variable does not have a statistically significant effect, then we can be certain that their behavior is conditioned by our experimental manipulation rather than their own preconceptions.

\section{Results of Regression Analyses Equality-Efficiency Preferences}

We analyze the equality-efficiency preferences of individuals using the Equality-efficiency tradeoff score described above as the dependent variable. Scores ranged from +30 (out of a possible +30 ), for the participant with the most extreme preference for equality, to -26 (out of a possible -30 ), for the participant with the most extreme preference for efficiency $(x=5.34, \mathrm{sd}=9.82)$. The independent variable of greatest interest is the between-subject experimental manipulation of merit, and we also include a variable for the poverty manipulation. For control variables we include dummy variables for female and minority, ${ }^{3}$ as well as variables for political ideology, mother's education (our measure of SES) ${ }^{4}$ and the participant's independent merit perception. Finally, to examine differences in response to the merit manipulation across gender and race we include interaction terms for merit and these dummy variables.

The results of our analysis of equality-efficiency preferences (Table 2A) show that the effects of the merit manipulation vary across participants, with a substantial difference between whites and minorities. Since a complete set of interaction terms is included in the regression equation, the unstandardized coefficient of -3.16

${ }^{3}$ We pooled all minority participants because a disaggregated analysis reveals almost no substantive differences among the subgroups. Additionally, we have no theoretical reason to expect different results across the subgroups. Pooling minority subgroups simplifies the analysis and increases its statistical power. We note the few cases of different results for minorities.

We tested three measures of SES and a factor of all three: mother's education, father's education, family income. We chose mother's education as the control variable because we have more complete data. The results do not change in significant ways if we use the other measures. 
TABLE 2 Results of Regression Analyses

\begin{tabular}{lcc}
\hline & \multicolumn{2}{c}{ Dependent Variable } \\
\cline { 2 - 3 } & $\begin{array}{c}\text { A } \\
\text { Equality-Efficiency Preferences } \\
\text { Equality-Efficiency Score }\end{array}$ & $\begin{array}{c}\text { Need Sensitivity } \\
\text { Need Sensitivity Score }\end{array}$ \\
\hline Independent Variables & & \\
Merit $(0,1,2)$ & $-3.16(.87)^{* * *}$ & $1.06(.62)^{*}$ \\
Poverty $(0,1)$ & & $-2.07(1.99)$ \\
$\quad$ Merit*Poverty & $.72(.78)$ & $-.17(.57)$ \\
Female & & $-1.12(.65)^{*}$ \\
$\quad$ Female* Merit & $3.72(1.25)^{*}$ & $-.32(.60)$ \\
Female*Poverty & $.25(.97)$ & $-1.13(.99)$ \\
Minority & & $.93(.85)$ \\
$\quad$ Minority* Merit & $-2.28(1.26)$ & $-.82(.57)$ \\
$\quad$ Minority* Poverty & $3.03(.95)^{* *}$ & $.92(.97)$ \\
Ideology $(0-4 ; 0=$ strong conservative $)$ & & $-.55(.37)$ \\
Ideology*Poverty & $1.22(.46)^{*}$ & $.28(.78)$ \\
Mother's Education $(0-5 ; 0=$ less than high school $)$ & & $-.49(.20)^{*}$ \\
$\quad$ Mother's Ed*Poverty & $.23(.25)$ & $.44(.31)$ \\
Independent Merit Perception $(0-100 \%)$ & & $-.02(.01)$ \\
Constant & $-.003(.02)$ & $13.38(1.49)^{* * *}$ \\
Adjusted R2 & $2.20(2.21)$ & .049 \\
$\mathrm{~N}$ & .075 & 600 \\
\hline
\end{tabular}

Note: ${ }^{*} \mathrm{p}<.05,{ }^{* *} \mathrm{p}<.01,{ }^{* * *} \mathrm{p}<.001$ : two-tailed test with OLS standard errors in parentheses for equality-efficiency preference analysis; one-tailed test with robust standard errors in parentheses for need sensitivity analysis.

for merit measures the effect of merit for white males. The merit manipulation has a statistically significant effect for white males in the expected direction, showing that they are increasingly willing to trade off equality for efficiency as merit level increases. Turning to females, the statistical significance of the dummy variable for females indicates that they are more egalitarian than white males, as predicted. The fact that the female*merit interaction term is not statistically significant indicates that females do not react differently to the merit manipulation from white males. Recalculating the coefficients to determine the effect of the merit manipulation on females alone $\left(2.91(.79)^{* *}\right)$ reveals that females on average are also increasingly tolerant of inequality as merit level increases. ${ }^{5}$

When we turn to minority participants, however, we find surprising results. As for equality-efficiency preferences, the fact that the minority variable is not statistically significant indicates that minorities on average

\footnotetext{
${ }^{5}$ In interpreting the interaction terms, we follow Friedrich (1982). In refiguring the results of the merit manipulation for females, we add the coefficient for merit $(-3.14)$ to the coefficient for the female*merit interaction term (.18) to produce the combined coefficient $(-2.96)$, and then recalculate the error terms in accordance with the formula Friedrich provides.

${ }^{6}$ In the results of the same analysis disaggregated by racial subgroup, blacks were statistically significantly less egalitarian than white males $\left(-3.23(1.72)^{*}\right)$. Results for other minority groups were not significant: Hispanics (-1.44 (1.60)), Asians (-1.37 (2.60)), other race $(-3.97(3.04))$.
}

have similar preferences to white males, and although the result is not quite statistically significant, the negative coefficient suggests that minorities are unexpectedly somewhat less egalitarian than white males. ${ }^{6}$ There are, however, evident differences between racial groups in the effect of merit on equality-efficiency preferences. Here the fact that the minority*merit interaction term is statistically significant indicates that minorities react differently to the merit manipulation than white males: namely, that their equality-efficiency preferences do not change across merit conditions. Supporting evidence suggests that minorities may be skeptical about claims that income outcomes are due to merit since in answering our post-experimental question about independent merit perception minorities gave considerably lower estimates than whites (although, as we note below, this variable is not itself a statistically significant predictor of equality-efficiency preferences). Whereas whites on average estimate the relationship between merit and income outcomes in the U.S. at $67.8 \%(\mathrm{sd}=19.3)$, minorities on average answer $61.3 \%(\mathrm{sd}=20.5)$. The difference is statistically significant.

As for the other variables, ideology is an important predictor of equality-efficiency preferences. As expected, liberals are more concerned with equality and conservatives with efficiency. SES does not show any effect. Finally, the fact that independent merit perception is not statistically significant in the analysis sug- 
gests that participants' behavior is explained by our experimental manipulation of merit rather than their own preconceptions.

\section{Need Sensitivity}

The results of our analysis of need sensitivity (Table 2B) also reveal considerable variation across participants, with gender an important predictor. The dependent variable is the Need sensitivity score, which measures participants' ratings of the income distributions affected by the poverty manipulation. Since the distributions affected by the poverty line manipulation are also the most unequal distributions, we must also include interaction terms for the poverty condition with all of the independent variables we test in order to distinguish the specific effect of the poverty manipulation on participants from their reactions to inequality. We use the same independent variables as in the previous analysis. ${ }^{7}$ For the same reason, we include interaction terms for merit and these independent variables and an interaction term for poverty*merit.

The main variable of interest for analyzing need sensitivity is the poverty factor, and, as in the previous analysis, that variable alone measures the effect of the distributions being in poverty for white males because of the interaction terms included in the equation. The effect of the poverty manipulation on white males is in the expected direction but it is not statistically significant (2.07 (1.99)). In turn, although the female* poverty interaction term is not statistically significant, the recalculated effect of the poverty manipulation for females is statistically significant in the predicted direction (3.20 $\left.(1.76)^{*}\right)$. Females on average therefore give lower scores to the income distributions affected by the poverty manipulation when poverty is an issue than when it is not. While the fact that females are more sensitive to need is consistent with our expectations, our results concerning race are surprising. Although the effect is in the predicted direction, the minority dummy variable is not statistically significant $(1.15(2.03)),{ }^{8}$ indicating that being a minority in itself has no effect on need sensitivity.

Turning to the other variables in the need sensitivity analysis, we see that the merit factor is statistically sig-

\footnotetext{
${ }^{7}$ We found heteroskedasticity across the error terms of the standard OLS regression. We therefore report robust standard error estimators using the Huber-White correction. The significance of the independent variables is the same for both ways of calculation.

${ }^{8}$ Only Asians show a response to the poverty manipulation $(-4.12$ $\left.(2.34)^{*}\right)$, and, like whites, give lower ratings to distributions affected by the poverty line when need is an issue. No other minority participants show a response to the poverty manipulation, but all are in the predicted direction: blacks (-.62 (2.27)), Hispanics (-.03 (2.22)), and other race $(-2.25(3.06))$.
}

nificant, indicating that white males are increasingly tolerant of inequality as merit level increases, consistent with our analysis of equality-efficiency preferences. The fact that the poverty*merit interaction term is not statistically significant indicates that their propensity to favor higher efficiency distributions does not significantly decrease even when poverty is at issue. The same is true for females as well as minorities. The SES proxy variable is statistically significant and negative, indicating less tolerance for inequality among lower-SES participants. The statistical significance of the SES* poverty interaction term, in turn, reveals that lower-SES participants are somewhat more sensitive to need independent of concern for inequality than higher-SES participants, in accordance with our expectations.

We were somewhat surprised at the overall weakness of the poverty manipulation on participants, particularly among minorities. Our results may also be due in part to a floor effect. Scores for the low-equality distributions were already so low on average that the added poverty condition had only a limited effect. For example, where the poverty line was set at $\$ 10,000,42 \%$ of participants gave the low-equality/high-efficiency distribution a rating of either 0 or 1 . The ratings of the other two distributions affected by the poverty line manipulation were similarly suppressed. We believe a concern for inequality masked any distinct sensitivity to need among at least some participants, which is consistent with a similar result in one experiment in our earlier study (Scott et al. 2001). We will return to these results in the discussion section.

\section{Categorizing Participants' Use of Allocation Principles}

Our between-subject regression analysis of equality-efficiency preferences reveals that participants vary in their use of both equality and efficiency as allocation principles, but this analysis does not enable us to determine whether participants are employing a distinct Rawlsian maximin strategy. We can examine this question through a within-subject analysis of participants' use of allocation principles where we compare their observed behavior to several alternative theoretical models.

\section{Theoretical Models of Participants' Use of Allocation Principles}

We develop alternative theoretical models of how individuals might prioritize allocation principles using tradeoffs posed by our matrix of income distributions (see Table 1). We restrict ourselves to models involving egalitarian, 


\section{FIGURE 2 Theoretical Models of Participants' Use of Allocation Principles}

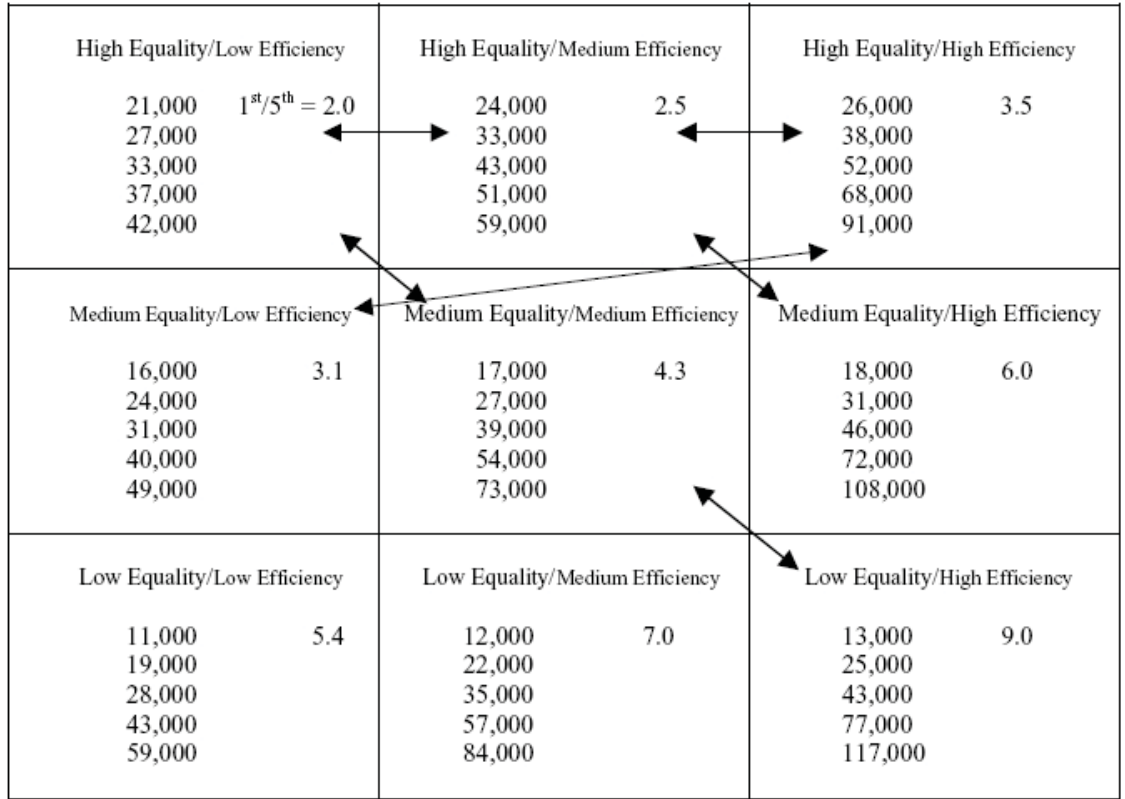

Strong Egalitarian

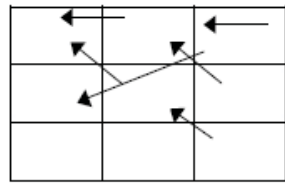

Strong Efficiency-Maximizer

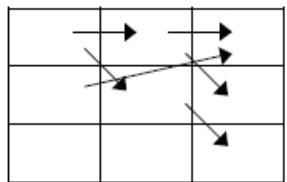

Moderate Egalitarian

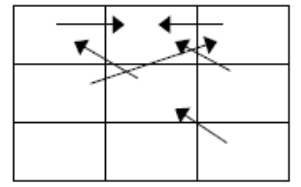

Moderate Efficiency-Maximizer

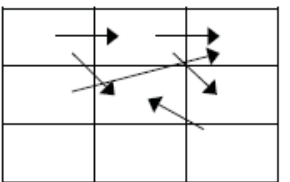

Mixed Equality-Efficiency

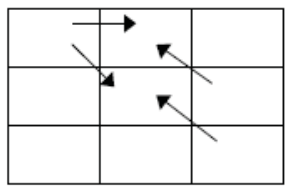

Rawlsian Maximin

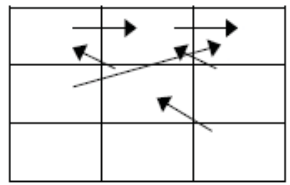

efficiency-maximization, and Rawlsian maximin strategies. In developing these models we balance the aim of using reasonably strict criteria of transitive preferences to distinguish between alternative models with the goal of capturing as much variance as possible. We therefore restrict our criteria to the more salient tradeoffs participants face and to those tradeoffs that best enable us to distinguish among the models.

The tradeoffs we use as criteria in constructing the theoretical models are represented by the arrows in the top figure in Figure 2. First, we use three of the four conspicuous diagonal tradeoffs used to construct the equality-efficiency score in our regression analysis. Each of these tradeoffs poses an obvious choice between a more equal but less efficient distribution and a less equal but more efficient one. We do not use the fourth diagonal trade-off (medium equality/low efficiency vs. low equality/medium efficiency) as a criterion because of the effect of the poverty manipulation on one of the distributions, but we do retain the other diagonal tradeoff affected by the poverty manipulation (medium equality/ medium efficiency vs. low equality/high efficiency) because it is particularly revealing of preferences for efficiency. Second, we use two horizontal tradeoffs in the high-equality row as criteria because they are particularly revealing of egalitarian preferences. Finally, we utilize one of the inverted diagonal tradeoffs as a criterion (medium equality/low efficiency vs. high equality/ high efficiency) since it poses a choice between egalitarian and Rawlsian maximin strategies. Whereas a Rawl- 
sian would prefer the efficiency gain in the high-equality/high-efficiency distribution because it maximizes the welfare of the least well-off, that is, the lowest income quintile, an egalitarian would have the opposite preference. We do not use the other inverted case (low equality/low efficiency vs. medium equality/high efficiency) as a criterion because of the effect of the poverty manipulation on one of the distributions. The remainder of Figure 2 presents a visual representation of the models we have developed, with the arrows indicating the direction of a participant's preferences in each of the tradeoffs we use as criteria.

The strictest models are the strong egalitarian and strong efficiency-maximizer models. The strong egalitarian model stipulates that a participant will prefer the more equal to the less equal distribution in each of the tradeoffs we use as criteria. In turn, the strong efficiencymaximizer model is essentially the reverse of the strong egalitarian model, with participants always preferring the more efficient distribution to the less efficient one in each of the tradeoffs.

The moderate egalitarian and moderate efficiencymaximizer models relax the most restrictive criteria used in constructing their stronger versions. The moderate egalitarian model follows the strong egalitarian model except for two changes. First, it relaxes one of the horizontal tradeoff criteria: a moderate egalitarian can have a preference for significantly greater income at the cost of slightly greater inequality, so they will prefer the highequality/medium-efficiency distribution to the highequality/low-efficiency one. Second, it relaxes the egalitarian criterion of the inverted diagonal case used to differentiate strong egalitarians from Rawlsians, stipulating that a moderate egalitarian will prefer the high-equality/ high-efficiency distribution to the medium-equality/lowefficiency one. The moderate efficiency-maximizer model similarly follows all of the criteria for the strong efficiency-maximizer model except in the diagonal tradeoff with the lowest equality levels, where a participant prefers the medium-equality/medium-efficiency distribution to the low-equality/high-efficiency distribution, whereas the opposite preference is stipulated for the strong efficiency-maximizer model.

The mixed equality-efficiency model preserves most of the criteria from the moderate egalitarian model while relaxing or eliminating others in order to allow for more tolerance for inequality than the moderate egalitarian model. First, we relax the most egalitarian among the three diagonal tradeoffs we have used as criteria, stipulating that a participant using this mixed strategy will prefer the medium-equality/medium-efficiency distribu- tion to the high-equality/low-efficiency one. We eliminate two other criteria we have otherwise used in order to maximize the variance we explain while retaining a fair degree of strictness in our criteria: the subjects in the mixed model are not acting in random fashion.

Finally, the Rawlsian maximin model is a mixed strategy model with several specific characteristics. A participant using a Rawlsian maximin strategy will maximize both equality and efficiency, but only on the condition of benefiting the least well-off. We therefore stipulate that, as in the egalitarian models, a Rawlsian will prefer the more equal to the less equal distribution in all three of the diagonal tradeoffs we use as criteria, because they are more equal and, more importantly, because the least well-off has a higher income level. On the other hand, for both of the horizontal tradeoffs the Rawlsian parts with the egalitarians in that she will prefer the more efficient distribution to the more equal one, because the least well-off are better off even though there is an increase in overall inequality. Lastly, we utilize the inverted diagonal tradeoff to distinguish strong egalitarians from Rawlsians, stipulating that a Rawlsian will prefer the high-equality/high-efficiency distribution to the medium-equality/low-efficiency one because the increased efficiency benefits the least well-off.

\section{Categorizing Observed Behavior}

In order to categorize participants' observed behavior according to the above theoretical models, we see if their behavior fits all of the criteria we establish for the different models. For each of the criteria, we determine whether their rating for the income distribution they should prefer is greater than or equal to the other distribution in the tradeoff. We begin our categorization with the strictest models, the strong egalitarian and strong efficiency-maximizer models. We then categorize the remaining participants using the moderate egalitarian then moderate efficiency models. Finally, we categorize those participants who remain using the mixed equality-efficiency and then Rawlsian maximin models. Using this procedure, we are able to categorize the observed behavior of $76.0 \%$ of participants.

Figure 3 reports the results of our categorization of participants' observed behavior using the theoretical models we have developed. ${ }^{9}$ The results are disag-

\footnotetext{
${ }^{9}$ The effect of using more restrictive theoretical models on the results of our categorization of participants' observed behavior is generally quite modest, but at the cost of not being able to categorize as many participants. For example, adding both the fourth diagonal tradeoff (medium equality/low efficiency vs. low equality/medium efficiency) and the second inverted diagonal tradeoff (low equality/low ef-
} 


\section{FIgURE 3 Categorization of Participants' Observed Behavior}

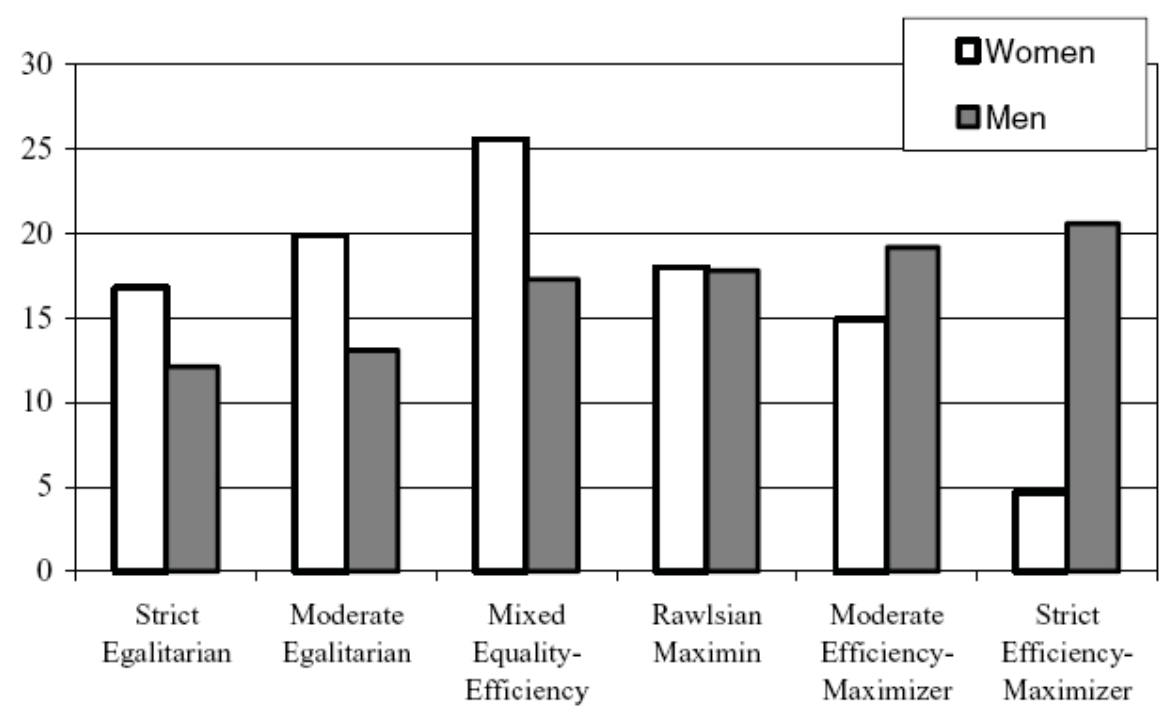

gregated by gender, which we found in our betweensubject analyses was the most important variable in explaining equality-efficiency preferences. Consistent with the results of the regression equations, we see a striking difference in the behavior of females and males regarding their use of egalitarian and efficiency-maximizing strategies. Females are more likely to be either strong or moderate egalitarians than males. Females are also more likely than men to use a mixed equality-efficiency strategy, which is still a relatively egalitarian strategy given the modest inequality of preferred outcomes. In turn, males are far more likely to be moderate efficiencymaximizers and especially strong efficiency-maximizers than females.

More importantly, our results reveal that a substantial number of participants $(18.0 \%)$ have behavior consistent with a distinct Rawlsian maximin strategy. There is no difference between females and males in terms of whether they are likely to use this strategy. That those participants fitting the Rawlsian maximin model are particularly sensitive to the benefit of the least well-off is further confirmed by comparing their ratings of the income distributions with the lowest income quintiles (the distributions in the low-equality level) with the ratings of participants fitting the other models. For this comparison

ficiency vs. medium equality/high efficiency) as criteria to all of the models except the mixed equality-efficiency model produces results very similar to those we report in the text, but with only $59.8 \%$ of participants categorized. With these added criteria, the proportion of Rawlsians among categorized participants remains almost exactly the same as with the models used in the text (17\% vs. $18 \%$ ), while the most significant change is a substantial reduction in those meeting the more restrictive strong egalitarian model ( $6 \%$ from $15 \%)$. we can utilize the Need sensitivity score used in the previous regression analysis. Those participants fitting the Rawlsian maximin model have a mean Need sensitivity score of 4.93 , while the mean scores of those fitting other models are substantially higher: strong egalitarian (8.76), moderate egalitarian (5.88), mixed egalitarian-efficiency (9.10), moderate efficiency-maximizer (7.75), and strong efficiency-maximizer (16.75). The differences between the mean scores for the Rawlsian maximin model and the other models are all statistically significant, except the moderate egalitarian model. ${ }^{10}$ This difference suggests that those participants fitting the Raw1sian maximin model are particularly sensitive to need, or the welfare of the least well-off. This result gives us good reason to believe that participants fitting the Rawlsian maximin model may be behaving not merely consistently with Rawls' theory, but acting as they do for the normative reasons Rawls specifies.

\section{Discussion}

Our study is a synthesis of normative and empirical research into distributive justice. We found that analytically distinct principles of distributive justice - equality, efficiency, merit, and need - all play a role in individuals' behavior when making judgments concerning income distribution and that several independent factors influ-

${ }^{10}$ We also analyzed participants' observed behavior using multinomial logistic regression taking the Rawlsian maximin model as the baseline, controlling for the uninteracted variables. We found no consistent statistically significant results not reported in the text, perhaps due to the low number of participants within each model once gender and race are taken into account. 
ence how they use these principles. The fact that most individuals use several allocation principles simultaneously makes their behavior necessarily complex, but this complexity is nonetheless comprehensible: both because the underlying theoretical relationship among the principles is structured and because the factors influencing how individuals use them are predictable. In short, distributive justice behavior is complex yet structured.

Before discussing our results, we want to address the possible limits to their generalizability. We used a convenience sample of undergraduates, as is the case in much experimental research, and this raises legitimate questions about external validity (see Sears 1986). We made several efforts to make our participant pool as diverse as possible: one test site, the University of Houston, is unusually ethnically diverse (over $50 \%$ minority) while the other, Texas Southern University, is a traditionally black institution. We cannot claim to have a maximally representative sample, nonetheless, foremost because our subject pool is skewed in terms of age. ${ }^{11}$ Survey data reveal that younger individuals are more egalitarian on average, and so it is plausible that a more representative sample would show participants less oriented to equality than our results suggest. Whatever the limits of external validity, however, our first concern was internal validity (see Kinder and Palfry 1993). The strength of experimental research is precisely internal validity, and we took several measures designed to promote such validity. First, we included a rigorous manipulation check to guarantee that only participants who received the experimental treatment and could properly interpret the information provided were included. Second, by asking participants in the post-experimental questionnaire about their own perceptions of the relationship between merit and income, and then entering that information as an independent variable in our regression analyses, we are able to determine that our experimental manipulations rather than participants' pre-existing perceptions explain our results. This test shows how using multiple regression analysis to enter additional controls can strengthen experimental research. Theses steps combined with the conformity of our results with theoretical predictions make us confident that we have strong internal validity and the basis to call for further research to confirm and extend these results.

\footnotetext{
${ }^{11}$ The mean age of our sample is 22 (min. 18, max. 55) $25 \%$ of the sample is over 22. A Kolmogorov-Smirnov test comparing our subjects' answers to questions in the post-experimental questionnaire taken from national surveys found no significant difference in similar age groups.
}

\section{Allocation Principles in Distributive Justice Judgments}

One of our aims in this study was to examine how individuals use allocation principles in distributive justice judgments, in particular to explore and extend the results of our study (Scott et al. 2001) under different experimental conditions. Our findings here generally confirm that previous research, but with some important clarifications and interesting new extensions.

Equality. We found considerable evidence that most individuals use equality as an allocation principle, even preferring more equal income distributions at the cost of a considerable loss in efficiency. This result confirms Scott et al. (2001), further reaffirms Mitchell et al.'s (1993) findings and also suggests that equality plays a stronger role than found by Frohlich and Oppenheimer (1992).

Efficiency. We found most individuals use efficiency as well as equality and try to strike a balance between the two principles. This result accords with Okun's (1975) influential framework for public policy analysis. Our findings concerning the factors that influence individuals' equality-efficiency preferences are also broadly consistent with Scott et al. (2001), and in particular underscore the strong gender difference they found. Likewise, we found that women tend to be more concerned with equality than men, and that men are more strongly oriented toward efficiency. We found no consistent differences between racial groups, although our results suggest that traditionally underprivileged minorities are no more egalitarian than whites and may even been somewhat more concerned with efficiency.

Merit. We found important results concerning the influence of gender and race on the way in which equality-efficiency preferences are mediated by assumptions about the relationship between merit and income outcomes. First, unlike the results of our earlier study (Scott et al. 2001), we found that both women's and men's equality-efficiency preferences are influenced by their assumptions about merit, although we found this result for white participants alone. In Scott et al. (2001) we unexpectedly but consistently found this to be the case for women alone. When discussing this result, we hypothesized that researchers might see the influence of merit assumptions among men if the cost of trading off equality for efficiency were increased and if a larger participant pool were employed. Our present study confirms this hypothesis. These combined results suggest that the relationship between merit assumptions and equality-efficiency preferences depends upon equality levels and 
the costs of the tradeoffs between the two principles and, further, that men and women tend to respond differently in these different contexts. Second, we found that race has a surprisingly strong influence on the way in which equality-efficiency preferences interact with information about merit. As noted above, the positive relationship we found between merit assumptions and equality-efficiency preferences held for whites of both sexes alone; information about merit did not affect the equality-efficiency preferences of minority participants. We will return this result concerning race below.

Need. Our poverty line manipulation was designed to examine a distinct concern for need and the factors that influence need sensitivity. We found that women showed a distinct sensitivity to need, but that men did not. We also found that white men are still concerned with merit at the highest levels of inequality and even with poverty an explicit issue, whereas women are instead affected by both the inequality of the distributions and independently by need. We suspect that the experimental design made it difficult to distinguish need sensitivity and intolerance for inequality, as was also the case for our earlier study (Scott et al. 2001). Finally, we found no statistically significant differences in need sensitivity between racial groups.

\section{Race and Gender in Distributive Justice Behavior}

Among the most significant findings in this study are those concerning race. Existing empirical literature gives very little, even conflicting, guidance in making predictions. We must also use some caution in interpreting our results on this subject in particular because we cannot claim to have a representative sample in spite of the size and diversity of our participant pool.

Our finding that race exerts an independent influence on how individuals use allocation principles calls into question the widely accepted-but not unchallenged-view in the existing behavioral literature that race has no strong independent predictive effect once one controls for other variables such as gender, ideology, and SES. Some of our results are consistent with this consensus, including our finding that minorities are no more sensitive to need than whites. On the other hand, we found clear differences between racial groups in how participants' equality-efficiency preferences were mediated by information about merit. When reporting this result, we conjectured that minority participants may be sensitive to information about merit because they appear to be suspicious about claims about the relationship between merit and income. We pointed as evidence for this conjecture to their significantly lower estimations of that relationship in our own society compared to whites. This finding, as well as the supporting evidence from the questionnaire, calls into question Hochschild's (1995) claim, based on interview and survey data, that blacks endorse the "American dream" concerning merit and socioeconomic mobility. Class identity (Jackman 1994) or racial group identity (Dawson 1994) appear to lead minorities to different views about distributive justice principles, and perhaps even different ways of thinking about them (see Kinder and Winter 2001). In sum, our study is the first study of which we are aware that suggests that race exerts an independent influence on distributive justice.

We also found considerable differences in how gender influences individuals' use of allocation principles, which suggest several reflections related to our findings regarding race. Consistent with previous research, we found that women are substantially more concerned with equality and more sensitive to need than men. Unlike our earlier study, (Scott et al. 2001), we did not find a gender gap in how merit assumptions affect equalityefficiency preferences, but we did confirm that the interaction among these principles is different for men and women in different contexts. Our findings concerning need in particular parallel findings regarding the different weights men and women give to social policy issues and suggest the same socioeconomic or other situational explanations which have been advanced for these results (Piven 1985; Gilens 1988; Welch and Hibbing 1992; Chaney, Alvarez, and Nagler 1998). In terms of our experiment, both women's and minorities' traditional socioeconomic position might explain why they weigh equality or need more heavily than men or whites. Likewise, their traditional vulnerability may explain why women tend to weigh equality more heavily than men, and why minorities appear suspicious of claims about merit. Our findings concerning gender and for race suggest we might look for explanations to the structural or situational differences in society between women and men and between whites and traditionally underprivileged minorities. In any case, our findings indicate a more complex theory and subtler instruments are necessary in studying gender and race differences in public opinion and behavior.

\section{Rawlsian and Other Strategies in Prioritizing Allocation Principles}

One of our principal aims in this study was to analyze strategies individuals use in prioritizing distributive prin- 
ciples, in particular to determine to what extent they use a Rawlsian maximin strategy. Our experimental design enabled us to develop several theoretical models for how individuals might prioritize allocation principles, which we then used to categorize their observed behavior. Our ability to capture the observed behavior of over threequarters of the participants further confirms that distributive justice behavior is complex yet structured.

We found that the observed behavior of a substantial number of participants is consistent with a Rawlsian maximin strategy - itself an important result for both normative and empirical research. This result is in opposition to the absence of support for Rawlsian solution found by Frohlich and Oppenheimer (1992) and tends to support Mitchell et al. (1993), although we believe that our study more clearly distinguished between egalitarian and Rawlsian strategies and therefore offers more compelling evidence. While we are prepared only to claim that some of our participants exhibit behavior consistent with Rawls' predictions, since we do not have access to their specific reasoning, supporting evidence concerning their particular sensitivity to the welfare of the least well-off suggests they may indeed be making judgments in accordance with the normative reasons Rawls provides. Further research is required to examine the characteristics of this subset of apparent Rawlsians and their reasoning. We should nonetheless not allow the support we find for Rawls to obscure the evidence that speaks against his theory. After all, the clear majority of participants in our study prioritized allocation principles in ways directly contrary to Rawls' prescriptions and most participants also clearly regarded merit as a legitimate moral principle, again contrary to Rawls' argument. Our findings concerning gender and racial differences in distributive justice behavior are similarly in some tension with Rawls since he assumes that such differences should not matter behind "the veil of ignorance." More consistent with our findings is Okin's (1989) argument that gender should be taken into account in the "original position." Rawls' theory of "justice as fairness" is one alternative among competing strategies to prioritize and balance potentially competing allocation principles.

\section{Conclusion}

Philosophers, politicians, and ordinary people alike are faced with complex normative and empirical questions concerning the distribution of the benefits and burdens of society. We should therefore not be surprised to discover that distributive justice behavior itself is complex. The complexity of distributive justice judgments is reflected in the most prominent contemporary theory of justice, Rawls' theory of justice as fairness, for it involves a specific ordering of the competing principles used in distributive justice judgments. Yet Rawls' endeavor and the results of our own study provide testimony to the structure of distributive justice behavior that lies beneath the complexity. Our study uncovers part of the complex structure of distributive justice judgments, and our findings should inform both normative and empirical inquiry. The fact that the principles used in distributive justice judgments are interrelated and that individuals' preferences concerning them are nonseparable, for example, suggests that survey research and other instruments must use a sophisticated design that reflects the true structure of these principles in order to produce accurate and valid results. We hope that our study shows the fruitfulness of a synthesis of normative and empirical approaches to distributive justice.

\section{Appendix}

Participant Instructions

Note: Portions in italics are varied between subject. The first selection specifies the degree to which income is explained by effort and ability $(10 \%, 50 \%, 90 \%)$, while the other selections specify the level of the poverty line ( $\$ 10,000$ and $\$ 15,000)$.

Assessment of Income Distributions. Please read the description below. Please ask if you have any questions at any time.

Imagine that you are an outside advisor to the country described below. You are not a member of the country. You are being asked to give your opinion on what policies would be best for the country. We are interested in your ideas about how income should be distributed when your own interests are not at stake.

Country D is a self-governed society made up of people of a variety of racial and ethnic backgrounds. There is a broad variety of occupations in the society. Scientific studies have demonstrated that the amount of income a person receives in Country D is due almost entirely to the person's effort and ability. People who are willing to work hard, take some risks, and acquire the proper education and skills have a very good chance of having a higher income. On the other hand, people who are not willing to do these things have a very good chance of having a lower income. The amount of income a person receives is $90 \%$ due to his or her effort and ability, and only $10 \%$ due to luck, connections, and similar factors.

The people of Country D are considering a number of policies for their society. Economic studies have dem- 
onstrated what effects the different policies will have on the average income in the country as a whole (standard of living) and on differences in people's income (income distribution). Backers of the different policies are convinced by these studies and do not disagree about the effects of the policies. They do disagree on which policy is the best for their country. Among other considerations, certain analysts are more concerned about the average income, while others are more concerned about equality of income.

You are being asked to give your opinion about how good the different policies would be for Country D based on their effects on income.

In order to assess these different policies, you will be presented with charts that show their effects on income. All the income figures you will be given are for after tax income for four-person families. All income figures are in U.S. dollar equivalents. The charts show family incomes broken down into each fifth $(20 \%)$ of families (or each "quintile"), from the poorest $20 \%$ of families in terms of income up to the wealthiest $20 \%$. The charts also give you information about the average income in the country as a whole and the income inequality ratio. The income inequality ratio is a commonly used measure of income inequality and is figured by dividing the income of the highest $20 \%$ of families by the income of the lowest $20 \%$ of families.

The chart below [see Figure 1] shows family incomes for Country D. As you can see, average family income after taxes are: $\$ 16,000$ per year for the poorest $20 \%$ of families, $\$ 26,000$ for the next $20 \%, \$ 34,000$ for the next $20 \%, \$ 53,000$ for the next $20 \%$, and $\$ 71,000$ for the wealthiest $20 \%$. The poverty line (the amount of income needed to provide for the basic needs of food, housing, clothing, and medical care for a four-person family) is $\$ 15,000$. The income inequality ratio is 4.4 (\$71,000 divided by $\$ 16,000)$.

\section{References}

Alwin, Duane F, Galin Gornev, and Ludmila Khakhulina. 1995. "Comparative Referential Structures, System Legitimacy, and Justice Sentiments." In Social Justice and Political Change, ed. James R. Kluegel, David S. Mason, and Bernd Wegener. New York: Aldine, pp. 109-30.

Braybrooke, David. 1987. Meeting Needs. Princeton: Princeton University Press.

Chaney, Carole Kennedy, R. Michael Alvarez, and Jonathan Nagler. 1998. "Explaining the Gender Gap in U.S. Presidential Elections, 1980-1992." Political Research Quarterly 51(June):311-39.

Daniels, Norman, ed. 1989. Reading Rawls: Critical Studies of
Rawls, “A Theory of Justice.” New York: Basic Books.

Davidson, Pamela, Susanne Steinmann, and Bernd Wegener. 1995. "The Caring but Unjust Women? A Comparative Study of Gender Differences in Perceptions of Social Justice in Four Countries." In Social Justice and Political Change, ed. James

R. Kluegel, David S. Mason, and Bernd Wegener. New York: Aldine, pp. 285-319.

Dawson, Michael C. 1994. Behind the Mule: Race and Class in African-American Politics. Princeton: Princeton University Press.

Elster, Jon. 1995. "The Empirical Study of Justice.” In Pluralism, Justice, and Equality, ed. David Miller and Michael Walzer. Oxford: Oxford University Press, pp. 81-98.

Friedrich, Robert J. 1982. "In Defense of Multiplicative Terms in Multiple Regression Equations." American Journal of Political Science 86: 4(3):797-833.

Frohlich, Norman, and Joe A. Oppenheimer. 1992. Choosing Justice: An Experimental Approach to Ethical Theory. Berkeley: University of California Press.

Gilens, Martin. 1988. "Gender and Support for Reagan: A Comprehensive Model of Presidential Approval." American Journal of Political Science 32(1):19-49.

Gilligan, Carol. 1982. In a Different Voice. Cambridge: Harvard University Press.

Hayek, Friedrich. 1976. Law, Legislation, and Liberty. Vol. II: The Mirage of Social Justice. London: Routledge and Kegan Paul.

Hegtvedt, Karen A., and Karen S. Cook. 2001. "Distributive Justice: Recent Theoretical Developments and Applications." In Handbook of Justice Research in Law, ed. Joseph Sanders and V. Lee Hamilton. New York: Kluwer Academic/Plenum, pp. 93-132.

Hochshild, Jennifer L. 1981. What's Fair? American Beliefs about Distributive Justice. Cambridge: Harvard University Press.

Hochshild, Jennifer L. 1995. Facing Up To the American Dream: Race, Class, and the Soul of the Nation. Princeton: Princeton University Press.

Jackman, Mary R. 1994. The Velvet Glove: Paternalism and Conflict in Gender, Class, and Race Relations. Berkeley: University of California Press.

Kinder, Donald R. and Thomas R. Palfrey. 1993. "On Behalf of an Experimental Political Science.” In Experimental Foundations of Political Science, ed. Donald R. Kinder and Thomas R. Palfrey. Ann Arbor: University of Michigan Press, pp. 1-39.

Kinder, Donald R., and Nicholas Winter. 2001. "Exploring the Racial Divide: Blacks, Whites, and Opinion on National Policy." American Journal Political Science 45(2):439-56.

Kluegel, James R., and Masaru Miyano. 1995. "Justice Beliefs and Support for the Welfare State in Advanced Capitalism." In Social Justice and Political Change, ed. James R. Kluegel, David S. Mason, and Bernd Wegener. New York: Aldine, pp. 81-105. 
Kluegel, James R. and Eliot Smith. 1986. Beliefs About Equality: Americans' Views of What is and What Ought to Be. Hawthorne, NY: Aldine de Gruyter.

Kluegel, James R., David S. Mason, and Bernd Wegener. 1995. Social Justice and Political Change. New York: Aldine.

Konow, James. 2000. "Fair Shares and Cognitive Dissonance in Allocation Decisions." American Economic Review 90 (September):1072-91.

Konow, James. 2001. "Fair and Square: the four sides of distributive justice." Journal of Economic Behavior and Organization 46(2):137-64.

Lacy, Dean. 2001. "A Theory of Nonseparable Preferences in Survey Responses." American Journal of Political Science 45(2):239-58.

Lane, Robert. 1962. Political Ideology. New York: Free Press.

Major, Brenda, and Jeffrey B. Adams. 1983. "Role of Gender, Interpersonal Orientation, and Self-Presentation in Distributive-Justice Behavior." Journal of Personality and Social Psychology 45(3):598-608.

Major, Brenda, and Kay Deaux. 1982. "Individual Differences in Justice Behavior." In Equity and Justice in Social Behavior, ed. Jerald Greenberg and Ronald Cohen. New York: Academic Press, pp. 43-76.

McClosky, Herbert, and John Zaller. 1984. The American Ethos: Public Attitudes Toward Capitalism and Democracy. Cambridge: Harvard University Press.

Miller, David. 1999. Principles of Social Justice. Cambridge: Harvard University Press.

Mitchell, George, Philip E. Tetlock, Barbara A. Mellers, and Lisa Ordónez. 1993. "Judgments of Social Justice: Compromises Between Equality and Efficiency." Journal of Personality and Social Psychology 65(4):629-39.

Nozick, Robert. 1974. Anarchy, State, and Utopia. New York: Basic Books.

Okin, Susan Moller. 1989. Justice, Gender, and the Family. New York: Basic Books.

Okun, Arthur. 1975. Equality and Efficiency: The Big Tradeoff . Washington, D.C.: Brookings Institute.

Ordónez, Lisa, and Barbara A. Mellers. 1993. "Trade-Offs in Fairness and Preference Judgments." In Psychological Perspectives on Justice: Theory and Applications, ed. Barbara Mellers and Jonathan Baron. Cambridge: Cambridge University Press, pp. 138-54.

Piven, Frances F. 1985. "Women and the State: Ideology, Power, and the Welfare State." In Gender and the Life Course, ed. Alice S. Rossi. New York: Aldine, pp. 265-87.

Rae, Douglas. 1981. Equalities. Cambridge: Harvard University Press.

Rasinski, Kenneth A. 1987. "What's Fair is Fair-Or is it? Value Differences Underlying Public Views About Social Justice," Journal of Personality and Social Psychology 53:201-11.

Rawls, John. 1971. A Theory of Justice. Cambridge: Harvard University Press.
Rawls, John. 1996. Political Liberalism. New York: Columbia University Press.

Rawls, John. 2001. Justice as Fairness: A Restatement. Cambridge: Harvard University Press.

Sandel, Michael. 1982. Liberalism and the Limits of Justice. Cambridge: Cambridge University Press.

Scott, John T., Richard E. Matland, Philip A. Michelbach, and Brian H. Bornstein. 2001. "Just Deserts: An Experimental Approach to Distributive Justice." American Journal of Political Science 45(3):749-67.

Sears, David O. 1986. "College Sophomores in the Laboratory: Influence of a Narrow Data Base on Social Psychology's View of Human Nature." Journal of Personality and Social Psychology 51(3):515-30.

Sen, Amartya. 1977. "Rational Fools: A Critique of the Behavioral Foundations of Economic Theory." Philosophy and Public Affairs 6(4):317-44.

Sen, Amartya. 1992. Inequality Reexamined. Cambridge: Harvard University Press.

Sen, Amartya. 1997. On Economic Inequality. Oxford: Oxford University Press.

Sher, George. 1987. Desert. Princeton: Princeton University Press.

Sniderman, Paul M., Michael Gray Hagen, Philip E. Tetlock, and Henry E. Brady. 1986. "Reasoning Chains: Causal Models of Policy Reasoning in Mass Publics." British Journal of Political Science 16(4):405-30.

Temkin, Larry S. 1993. Inequality. Oxford: Oxford University Press.

Törnblom, Kjell Y. 1992. "The Social Psychology of Distributive Justice." In Justice: Interdisciplinary Perspectives, ed. Klaus R. Scherer. Cambridge: Cambridge University Press, pp. 177-236.

Tyler, Tom R., Robert J. Boeckmann, Heather J. Smith, and Yuen J. Huo. 1997. Social Justice in a Diverse Society. Boulder: Westview. Verba, Sidney, and Orren, Gary R. 1985. Equality in America: The View from the Top. Cambridge: Harvard University Press.

Verba, Sidney, Steven Kelman, Gary R. Orren, Ichiro Miyake, Joji Watanuki, Ikuo Kabashima, and G. Donald Ferree, Jr. 1987. Elites and the Idea of Equality: A Comparison of Japan, Sweden, and the United States. Cambridge: Harvard University Press.

Walzer, Michael. 1983. Spheres of Justice: A Defense of Pluralism and Equality. New York: Basic Books.

Weatherford, M. Stephen. 1992. "Measuring Political Legitimacy." American Political Science Review 86 (March):14966.

Welch, Susan, and Hibbing, John. 1992. "Financial Conditions, Gender, and Voting in American National Elections." Journal of Politics 54 (February):197-213. 